\title{
Speed estimation during the starting transient of induction motors
}

\author{
Matias Meira, Student Member, IEEE, Guillermo R. Bossio, Senior Member, IEEE, Carlos J. \\ Verucchi, Cristian R. Ruschetti, Member, IEEE, and José M. Bossio, Member, IEEE
}

\begin{abstract}
Typically, the rotor speed of electric motors was measured directly by the use of electromechanical sensors. Even though these devices are very precise, they are also fragile and expensive to install. Currently, some alternatives are based on speed estimations from the measurement of stator currents. Some of these, very accurate, are used in variable speed drives. In industrial power applications, many large induction motors are directly driven with special starters. For these cases, new speed estimation strategies must be developed. This paper presents a self-sensing method for speed estimation during the starting transient of both wound rotor and squirrel cage induction motors. The speed estimation is based on the simultaneous tracking of multiple harmonic components of the rotor on the spectrogram of the stator currents in sequence networks. The proposal is validated with experimental results obtained in the laboratory with a squirrel cage induction motor. It is concluded that the estimation is not sensitive to measurement noise and tracking errors caused by other harmonic components that do not depend on the rotor position.
\end{abstract}

Index Terms - induction motor; starters; speed estimation; diagnosis.

\section{INTRODUCTION}

$\mathrm{T}$ $\mathrm{O}$ avoid line overloads, large voltage drops, and thermal stresses during the starting transient of high power induction motors (IMs), the use of starting methods is essential. From $5.5 \mathrm{~kW}$ onwards, starting methods at a reduced voltage or starting by static converters are used. Direct starting is allowed only for minor power. If high starting torque is required, reduced voltage starting is not viable since the starting torque decreases with the square of the voltage. Therefore, start-ups with rotor resistance variation are necessary.

Knowing the rotor speed is not only useful as feedback in variable speed drives, but also as analysis and diagnostic tool [1]. For example, if the instantaneous rotor speed and the

M. Meira, INTELYMEC (UNCPBA) and CIFICEN (UNCPBA-CICPBACONICET), Olavarría, Argentina (e-mail: matias.meira@fio.unicen.edu.ar).

G. R. Bossio, GEA-IITEMA-UNRC-CONICET, Río Cuarto, Argentina (email: gbossio@ing.unrc.edu.ar)

C. J. Verucchi, INTELYMEC (UNCPBA) and CIFICEN (UNCPBACICPBA-CONICET), Olavarría, Argentina (e-mail: verucchi@fio.unicen.edu.ar).

C. R. Ruschetti, INTELYMEC (UNCPBA) and CIFICEN (UNCPBACICPBA-CONICET), Olavarría, Argentina (e-mail: cruschet@fio.unicen.edu.ar).

J. M. Bossio, GEA-IITEMA-UNRC-CONICET, Río Cuarto, Argentina (email: jbossio@ing.unrc.edu.ar) torque transmitted to the load are known, it is possible to estimate the operating point in relation to the torque-speed curve provided by the IM manufacturer. If in permanent regime, the operating point does not coincide with a point on the curve, an anomaly is evidenced (such as voltage imbalances, high harmonic content in the supply voltage, or load imbalances). Furthermore, the starting conditions of an IM can be verified through the rotor speed. For example, if the starting times are kept below the limits indicated by the manufacturer, or if the resistances of liquid starters are adequate or the ramp settings in soft starters ensure the best operating point [2]. Direct speed measurement by electromechanical speed sensors in industrial environments is not always possible since these sensors are fragile. In this situation, sensorless self-sensing methods for speed estimation gain notoriety. Table I presents some of the strategies reported in the literature and highlights purposes, ranges of application, and errors among other factors of interest.

Many rotor speed or position estimation strategies have been presented focused on control of variable speed drives. There are two main categories of sensorless control schemes: model-based and saliency-based [3]. Model-based strategies are applied in the high-speed range. Among these approaches, the use of observers or parametric methods for this purpose is widespread. Observers are sensitive to parameter variation or detuning. To improve their performance, the use of an optimized constant rate reaching law was proposed in [4]. In [5], a strategy to identify parameters independent of the speed observer's output through flux estimation is proposed. With speed estimation strategies that are not very sensitive to multiparameter variations, the performance of the observers is improved, and therefore, the speed estimation in steady state and transient conditions [6]. However, observers require motor parameters and are difficult to apply if these parameters vary during starting. This is the case, for example, with star-delta or variable rotor resistance starting in IMs. Saliency-based strategies are applied in the low-speed range. Many of these techniques are based on high frequency signal injection. As an example, [7] uses transient voltage pulses excitation for speed estimation by the discrete cosine and sine transforms (DCT and DST). These signals can easily be generated by the inverter of the drive but are difficult to implement in IMs connected directly to the grid. Henceforth, these strategies cannot be applied to all industrial cases, where there are direct drives. For this reason, it is 
TABLE I Comparison OF Different Speed Estimation Strategies, PuRPoses AND ERRORs

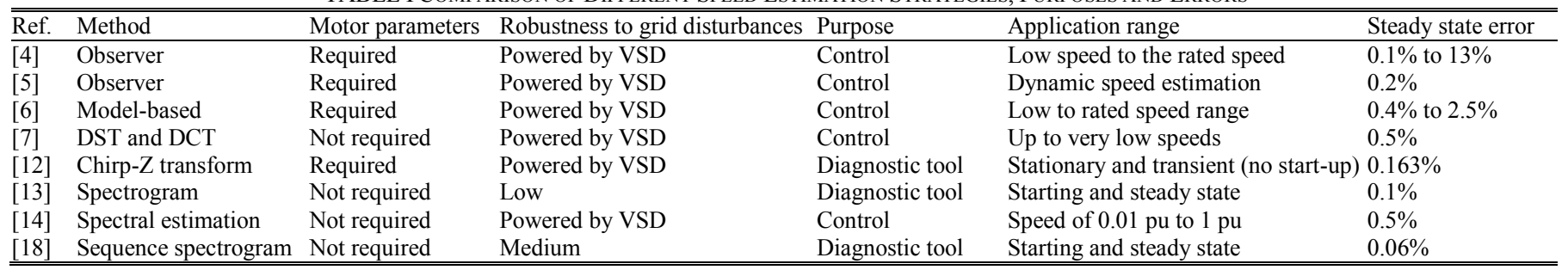

necessary to develop speed estimation alternatives for these cases. Here, the estimation by frequency tracking of the rotor harmonics present in the stator current, associated with the rotor position, becomes promising. In this line, [8] introduces a speed sensorless method for detecting rotor asymmetries in wound rotor induction machines working under non-stationary conditions using the Gabor transform. Broken rotor bars throughout the synchrosqueezing wavelet transform [9], bearing faults and rotor eccentricity throughout the Fourier synchrosqueezing transform [10] can be detected by the use of frequency components originated in the rotor and reflected in the stator currents during the starting transient. Electromechanical torque, obtained from the speed estimation and the active power consumed by the IM, can provide useful information for the diagnosis of motor faults and their efficiency [11].

IM speed estimation from the tracking of certain harmonic components has been presented in the literature backgrounds [12], [13]. Harmonics present in the stator current, linked to the number of rotor slots, are the most used for this purpose [14]. This methodology is robust when the frequency of the supply voltages and the load resistant torque are constant [15], [16]. The accuracy of this technique depends directly on the precision with which it is possible to identify the harmonics associated with the rotor slots. In this sense, [17] provides proper guidelines for the selection of stationary and timevarying harmonic and interharmonic estimation methods according to a given application.

In [18], a self-sensing method for the IM speed estimation during the starting transient, based on the tracking of a single harmonic component of the rotor on the spectrogram of the stator currents, was presented. In this work, as an extension of that proceedings paper, a simultaneously tracking of multiple harmonic components of the stator currents full spectrogram is proposed, which allows obtaining less sensitive speed estimation to measurement noise and tracking errors.

\section{HARMONIC COMPONENTS PRESENT IN THE IM}

IMs presents sinusoidal rotor and stator currents if it is perfectly balanced and supplied with balanced voltages, and its magnetic field rotates at a frequency $f_{l}$ in the stator reference frame or $s f_{l}$ in the rotor reference frame. Where $f_{l}$ is the supply voltage frequency and $s$ is the IM slip.

However, certain constructive characteristics or imperfections determine the presence of harmonic components in both rotor and stator currents even in a healthy IM [16], [19]. In Table II, it can be found the most used expressions in practice to identify the frequencies of the harmonic components generated in the stator currents by different constructive characteristics of IMs or those generated by faults. $k$ is a positive integer $(0,1,2,3,4,5, \ldots), R$ is the number of rotor slots, $n_{d}$ is an integer $(0, \pm 1, \pm 2, \pm 3, \pm 4, \pm 5 \ldots), P$ is the number of pole pairs and $n_{w}$ is the harmonic order $( \pm 1, \pm 3$, $\pm 5, \pm 7, \pm 9, \ldots)$.

TABLE II

HARMONIC COMPONENTS FREQUENCIES IN THE STATOR CURRENTS

\begin{tabular}{lll}
\hline \hline \multicolumn{1}{c}{ Ref. } & Characteristic & \multicolumn{1}{c}{ Frequency } \\
\hline$[20]$ & Rotor asymmetry & $f_{b}=f_{l}(1 \pm 2 s)$ \\
{$[21]$} & Eccentricity & $f_{e c c}=f_{l}\left[1 \pm \frac{(1-s)}{P}\right]$ \\
& & $f_{c o m b-e c c}=f_{l}[1 \pm(1-s)]$ \\
& & $f_{s h}=f_{l}\left[\left(k R+n_{d}\right)\left(\frac{(1-s)}{P}\right)+n_{w}\right]$ \\
\hline 120$],[15]$ & Rotor slots & \\
\hline
\end{tabular}

\section{DESCRIPTION OF THE SPEED ESTIMATION STRATEGY}

As can be seen from the previous section, the harmonic components from (1) to (4) are a function of slip, that is, they are a function of the rotation speed. Despite these components can be associated with machine faults, they are present in all IM due to the asymmetries inherent in the construction process, even in those that can be considered fault-free [19].

Therefore, it is possible to use one or more of these harmonic components for speed estimation. The proposed strategy uses the rotor slots harmonics present in the waveform of the stator current, given by (4).

A practice limitation, although it is shown that the frequency calculated with $k=1$ and $n_{d}=0$ can be distinguished in most cases (even in fault-free motors) [15], is that its value still depends on parameters such as the number of slots, which is generally unknown. So, the frequencies related to the slot harmonics change from one IM to another, making it difficult and impractical to calculate these frequencies. However, by processing the signal to be analyzed and subsequent simple visual inspection, it is possible to identify these frequencies. The proposed strategy focuses on this concept, analyzing the stator currents through sequence networks [22].

Once the frequency components of the different harmonics have been identified, it is possible to calculate the rotation frequency corresponding to each of them $\left(f_{r}\right)$, and finally the rotor speed, according to:

$$
n=\frac{60 f_{r}}{P}
$$

As can be seen from (1) to (4), a large number of harmonics arises that can be followed to estimate speed, whether positive or negative, so it is possible to obtain different speed estimations. Therefore, it is probable that not all estimates are 
coherent neither coincident with each other. The selection criteria will be detailed in subsection III.F.

\section{A. Filtering of fundamental and power grid harmonics}

Eliminate both the fundamental component of the power grid and its harmonics from the stator current is advantageous to track the harmonic components of the rotor associated with its speed. The harmonic components of the electrical grid have larger amplitudes than the harmonics produced by the rotor and they are generally found at very close frequencies. This can generate errors in the speed estimation strategy, so it is convenient to filter the signal to be analyzed. The most commonly used filters in the frequency domain are the comb filters and the multirate filters, which eliminate harmonic interference [23]. In this work, a notch-type comb filter was used. The notches are found every $f_{l}(50 \mathrm{~Hz})$, filtering the fundamental component of the electric grid frequency and its harmonics.

Furthermore, a second-order Butterworth low pass filter with a high cutoff frequency $(1000 \mathrm{~Hz})$ is applied to the analyzed signals in order to eliminate the high frequency noise present. A review of the different techniques for the suppression of the fundamental component is presented in [24], as well as new techniques for this purpose.

\section{B. Short Time Fourier Transform: spectrogram}

To perform an analysis of non-stationary periodic signals, in which their characteristics vary according to time, it is necessary to study them in the time-frequency domain. This is the case when analyzing the stator currents in the starting transient of IMs. A tool widely used to analyze this type of signals is the Short Time Fourier Transform (STFT), defined as [25]:

$$
X(\omega, \tau)=\int_{-\infty}^{\infty} x(t) f(t-\tau) e^{-i \omega t} d t
$$

where $x(t)$ is the analyzed signal and $f(t-\tau)$ is a window function around time $\tau$.

From the analysis of the spectrogram, in which the magnitude of the STFT is represented as a function of $\omega$ and $\tau$, the positive and negative harmonic components of the rotor linked to its position were extracted.

The STFT extracts several frames of a signal that are analyzed in each of the window displacements over time, considering the signal during this frame is stationary. Therefore, in each frame, it is possible to use the Fast Fourier Transform (FFT), where a continuous spectrum of a periodic signal is assumed.

The spectral resolution to be achieved depends on the number of points used to perform the FFT (nFFT). If it is wanted to have a minimum frequency resolution $f_{\text {ResMin }}$ from a fixed sampling frequency $F_{s}$, the nFFT number is determined as $F_{s} / f_{\text {ResMin }}$. Besides, it is highly recommended that $\mathrm{nFFT}$ be a power of two since in this way the FFT performs faster. However, increasing nFFT beyond the number of samples in the window will just append zeros to the end so it is the correct size (zero padding). This can be problematic if the signal is not close to zero at the beginning and the end, therefore the Hanning window is used. For this reason, nFFT should be close or slightly higher than the number of samples in the window. This determines the temporal length of the window, that is, the $\Delta t$.

Accordingly, for obtaining a high quality spectrogram, it is crucial a suitable selection of the lattice parameters $\Delta t$ and $f_{\text {ResMin }}$. This generates uncertainty in the time-frequency plane when a frequency variation occurs. This is because the resolution depends on the size of the window chosen for the analysis. Larger windows allow precise resolution in frequency; narrow windows allow correct temporal location. A compromise between temporal or frequency uncertainty has to be established, which will depend on each type of application.

The use of the Hanning window also fulfills the integer number of cycles requirement of the measured signal to perform the FFT. If this is not fulfilled, the spectrum obtained is a distorted version of the actual spectrum of the original signal. Therefore, window functions are used to overcome this drawback. These functions multiply the temporal signal acquired with a signal of the same length whose amplitude varies from a maximum value in the center to zero at the edges.

Nevertheless, the use of non-rectangular windows to reduce spectral leakage almost always decrease to zero at boundaries, so some data is lost. To retrieve that, the overlapping of the windows is carried out when processing. A $50 \%$ overlap is widely used to retrieve data between windows.

In this work, besides the time-frequency analysis through the STFT, the Hanning window was used for the analysis of the stator currents.

\section{Sequence components}

According to Fortescue's theorem, three unbalanced phasors can be represented by three systems of three balanced components; positive, negative, and zero (homopolar) sequence. Since IMs usually do not have neutral grounding, only positive and negative sequence components are established. For its calculation, the measurement of two of the three IM stator currents is required [22]. This strategy is quite similar to that used in vibration analysis to obtain the full spectrum of two quadrature signals [26]. The full spectrum can also be obtained by performing the complex FFT to quadrature currents as proposed in [27]. The full spectrum combined with the spectrogram function allows tracking the temporal evolution of the sequence components of the stator current. As the network harmonics are not related to the position of the rotor, its filtering facilitates the tracking of those that are linked.

\section{Speed estimation using single harmonic}

As it was mentioned above, the speed estimation is based on the tracking of rotor harmonics present in the stator current. For this, the spectrogram is calculated in sequence components for the filtered stator currents. Once the spectrogram is obtained, the harmonic components related to the corresponding load state of the IM speed are identified. 
The tracking of these components is carried out following the spectrogram in the opposite direction to time. This requires establishing a search frequency range around the center frequency identified in the permanent regime. This range has a central frequency and a $\Delta f$, which can be asymmetric. This asymmetry is because when the spectrogram is traveled in the opposite direction to time, the rotor speed decreases. The frequency corresponding to the maximum amplitude within the frequency range is selected, being this the new central frequency. Such central frequency displaces the search band, centering it with respect to the maximum amplitude value, thus allowing finding the frequencies related to the rotation speed. The harmonic frequencies found are linked to the rotation frequency by (4) if the harmonic is of positive sequence or by (5) if the harmonic is of negative sequence.

$$
\begin{aligned}
& \omega_{r}=2 \pi\left(f_{l}-\frac{\left|n_{w}\right| f_{l}-f_{r p s}}{k\left|n_{w}\right| \pm 1}\right) \\
& \omega_{r}=2 \pi\left(f_{l}-\frac{\left|n_{w}\right| f_{l}+f_{r n s}}{k\left|n_{w}\right| \pm 1}\right)
\end{aligned}
$$

where $\omega_{r}$ is the rotation frequency of the rotor in $\mathrm{rad} / \mathrm{s}, f_{r p s}$ and $f_{r \text { ns }}$ are the harmonic sequence components used for the estimation of the rotation frequency in $\mathrm{Hz}$, with $k \neq 0$.

Applying (5) to each $\omega_{r}$ found the rotation speed for each harmonic is determined.

\section{E. Speed estimation using multiple harmonics}

Instead of using a single harmonic to estimate the speed in the starting transient [18], it is also possible to simultaneously track multiple harmonic components of the stator currents full spectrogram $Y(\omega, \tau)$. Thus, it is possible to obtain estimation less sensitive to measurement noise and tracking errors caused by other harmonic components that are not linked to the rotor position. $Y(\omega, \tau)$ is the amplitude of the spectrogram in sequence components of the stator currents where the fundamental and its harmonics have already been filtered through the comb and low pass filter.

This estimation strategy proposes to track multiple harmonics $\omega_{j}$ that depend on rotor speed:

$$
\omega_{j}=f_{j}\left(\omega_{r}\right)
$$

where $j=1,2 \ldots n$, and $n$ is the number of harmonics to track.

To simultaneously find the maximums of each component of the spectrogram at every time $\tau_{i}$, the following objective function is used:

$F_{i}(\omega)=\alpha_{1} \frac{Y\left(\omega_{1}, \tau_{i}\right)}{Y_{1 f}}+\cdots+\alpha_{j} \frac{Y\left(\omega_{j}, \tau_{i}\right)}{Y_{j f}}+\cdots+\alpha_{n} \frac{Y\left(\omega_{n}, \tau_{i}\right)}{Y_{n f}}$

where $\omega_{\text {ri min }} \leq \omega \leq \omega_{\text {ri max }}$ and $\alpha_{j}$ are weights assigned to each of the frequency components $\omega_{j}=f_{j}(\omega)$. To normalize in amplitude the harmonic components, $Y_{j f}$ is the amplitude of the component at $\omega_{j}$ for the final time of the transient (IM in steady state):

$$
Y_{j f}=Y\left(\widehat{\omega}_{j}, \tau_{f}\right)
$$

Then the rotor speed is determined by looking for the maximum of $F_{i}(\omega)$ as (12).

$$
\left(F_{\operatorname{maxi}}, \widehat{\omega}_{r i}\right)=\max F_{i}(\omega)
$$

In this strategy, in addition to the speed estimation method, adaptive frequency search bands were implemented, which are reduced linearly with the estimated rotor speed ( $\Delta f$ is asymmetrical and of decreasing value). This achieves a further reduction in error at low estimated speeds since the interference between different harmonic components is constrained.

Fig. 1 shows the general procedure used for speed estimation for any method. Fig. 2 shows the recursive algorithm for speed estimation using multiple harmonics as an extension to the block "Speed estimation algorithm: single or multiple harmonics" from Fig. 1.

\section{F. Confidence criteria for estimated speed}

In most field cases, direct measurement of rotor speed is not available. Therefore, to verify the accuracy of the estimator from a temporal point of view in relation to the current it is necessary to establish some confidence criteria. These criteria are:

- In a steady state, the speed must be the corresponding one for the load factor in which the IM is operating. For this, the electromechanical torque could be estimated, which requires the measurement of the voltage; through current, linearizing the operation zone and knowing the no-load current, among other alternatives.

- The estimated speed must be zero before the starting transient. This implies that the estimated speed must be correlated with the current.

These confidence criteria apply to both estimated speeds from the use of single harmonics and multiple harmonics. In the case of using single harmonics, more than one curve may simultaneously meet the two conditions mentioned above. For that particular case, it is very likely that the curves coincide or are very close to each other, being able to select one of them without making too much error. In case this is not fulfilled, the comb filter may need to be adjusted better to reduce the effects of grid harmonics or the measured signal is too noisy.

\section{G. Scope of the proposed strategy}

The speed self-sensing strategy is off-line and applies to all slow start transients (the limit of the speed ramp depends on the order of the harmonics tracked and the number of pole pairs of the motor), where the IM is loaded with large inertias (the estimation is not made in real time). Its purpose is to diagnose problems in starters, coordinate protections, or switching times. Its limitation is that if the STFT time window is comparable with the starting transient duration, the speed estimation could not be performed. This occurs in the case of fast transients (speed ramp with a rate greater than $500 \mathrm{rpm} / \mathrm{s}$ ). However, the method applies to both squirrel cage and wound rotor IM and the transients in large power applications are generally slow (in the case of medium voltage wound rotor induction motors with liquid starters, speed ramp rates are between $30 \mathrm{rpm} / \mathrm{s}$ and $50 \mathrm{rpm} / \mathrm{s}$ ).

Besides, the strategy does not require the presence of frequencies originated by faults since it is based on the tracking of harmonic components present in all IM, regardless of whether they are fault-free or not, a necessary condition for other estimation methods [13]. 


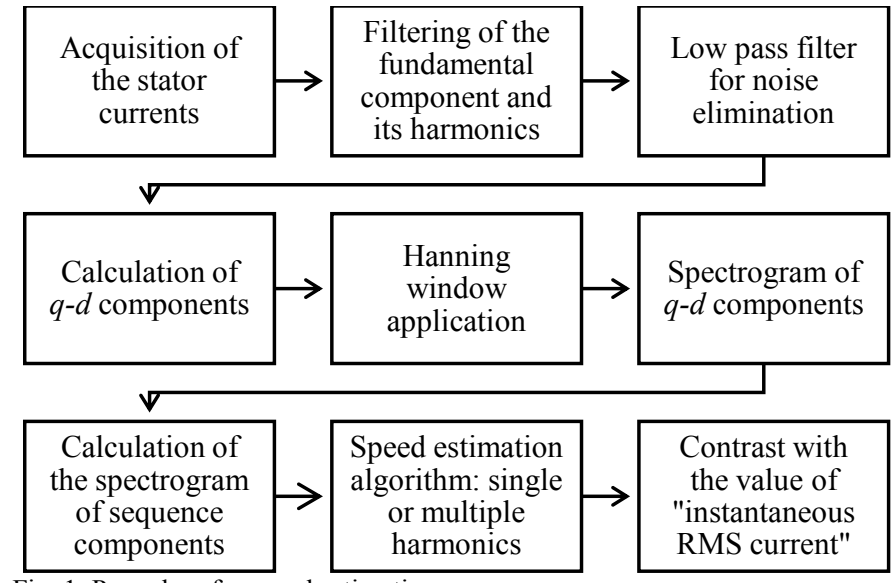

Fig. 1. Procedure for speed estimation.

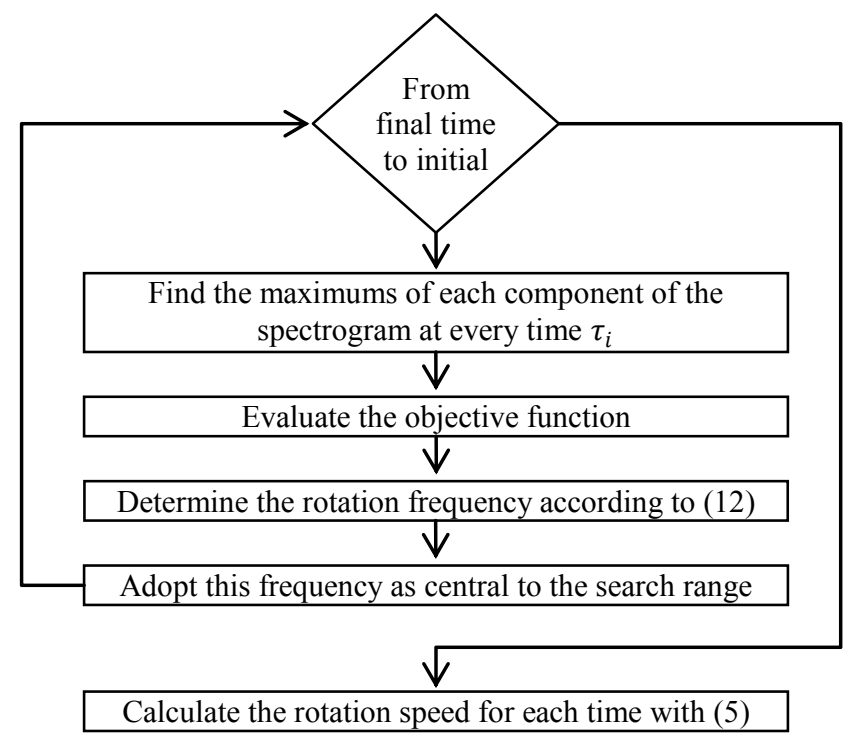

Fig. 2. Recursive speed estimation algorithm. Multiple harmonics approach.

For this case study, a frequency resolution of $2 \mathrm{~Hz}$ and a window length of $0.5 \mathrm{~s}$ were adopted as it is considered sufficient. With this setting, the range of rotor speed variation is contained in the spectrogram resolution so it can be estimated. The resolution of the estimated speed is $k\left|n_{w}\right| \pm 1$ times higher than that of the spectrogram. This is obtained by calculating a $\omega_{r}$ differential of (7) or (8). Particularly, the resolution of the estimated speed is 14 times higher than $2 \mathrm{~Hz}$ $(0.1428 \mathrm{~Hz})$.

\section{EXPERIMENTAL RESULTS}

Experimental tests were carried out on a squirrel cage IM, $5.5 \mathrm{~kW}, 380 \mathrm{~V}, 4$ poles, $1450 \mathrm{rpm}$, coupled to a DC generator as load. The speed estimation using multiple harmonics was put under test for its novelty and advantages over the single harmonics approach.

Two starting conditions were analyzed, one at reduced voltage and the other with a star-delta starter. For both cases, the sampling frequency of the stator currents was $10 \mathrm{kHz}$. The number of harmonics to track for the objective function (10) was three: the components around $\omega_{1}=5 f_{l}, \omega_{2}=7 f_{l}$, and $\omega_{3}=$ $13 f_{l}(250 \mathrm{~Hz}, 350 \mathrm{~Hz}$, and $650 \mathrm{~Hz}$ respectively).
To calculate the spectrogram in sequence components, the signal was divided into 50 sections of equal length, with a $50 \%$ overlap between sections. Thus, a resolution of $2 \mathrm{~Hz}$ is obtained in the spectrogram of the sequence components of the stator current and a resolution of $0.1428 \mathrm{~Hz}$ for the rotor frequency estimation.

\section{A. Speed estimation using both single and multiple harmonics at reduced voltage starting}

For this test condition, a $70 \mathrm{~V}$ line voltages starting was made and acquired. The only load for the motor was the inertia of the generator. Thus, a gradual starting of $16 \mathrm{~s}$ was achieved. The parameters of the comb filter were adjusted to achieve optimum filtering of the grid harmonics.

Fig. 3 shows the spectrogram of the filtered current in sequence components and the harmonics tracked. The search bands are not shown since both methods track the harmonics in the same way. Likewise, it is achieved that the speed estimation by both single or multiple harmonics is similar, as seen in Fig. 4.From Fig. 5, the speed error is less than 6.6\% during almost the whole starting transient and $0.04 \%$ during the steady state for the worst performing estimator.

From this case study, it can be concluded that with correct signal filtering and low noise content, both strategies have the same performance. However, in field cases, where there is no direct speed measurement or there is noise present in industrial facilities, the high dependence on the adjustment of the comb filter makes the estimation less reliable.

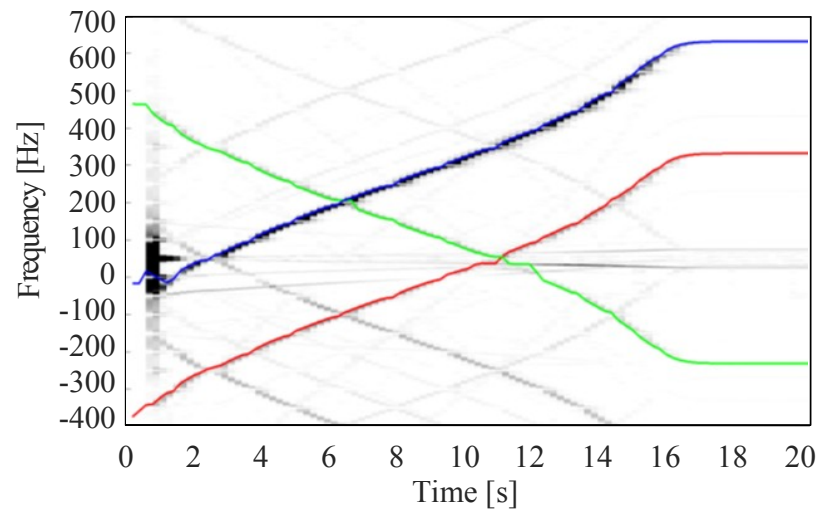

Fig. 3. Spectrogram of the sequence components of the filtered current for reduced voltage starting with fine adjustment of the comb filter.

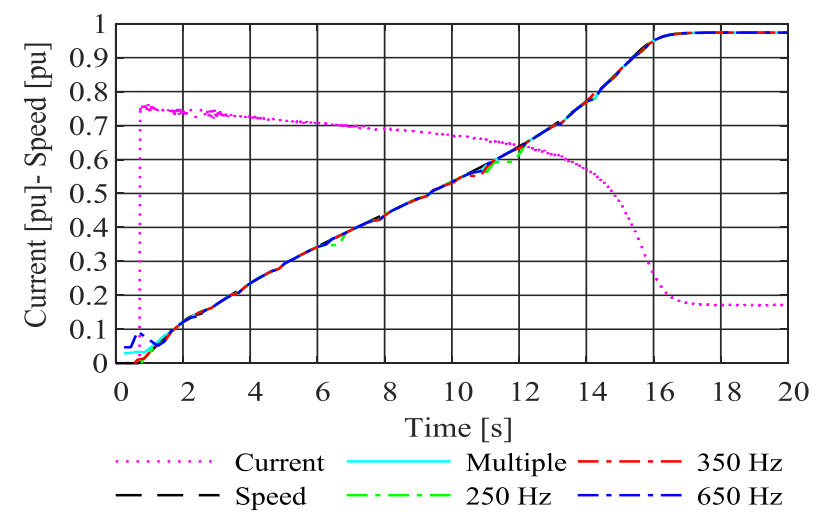

Fig. 4. Estimated speed and measured speed for reduced voltage starting with fine adjustment of the $c o m b$ filter. 


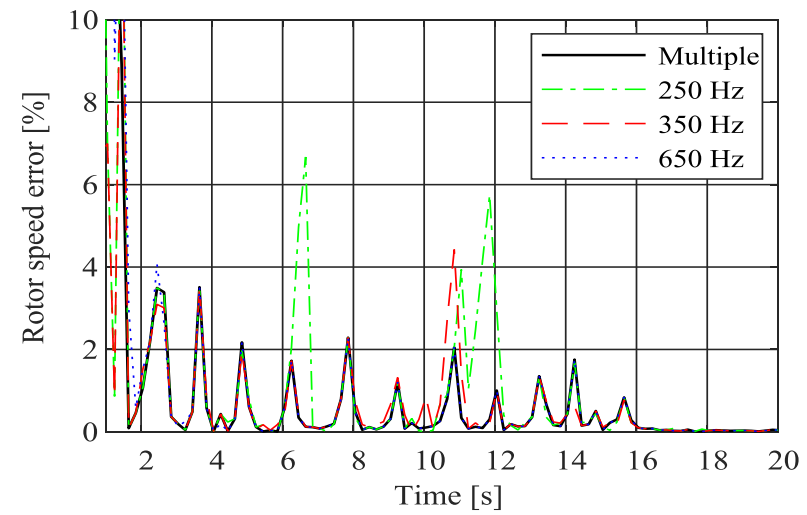

Fig. 5. Speed estimation error during starting transient for reduced voltage starting with fine adjustment of the comb filter.

\section{B. Speed estimation using single harmonics at reduced voltage starting}

For this case study, the same acquisition as the previous case was used with the exception that the comb filter does not completely eliminate the grid harmonics. Its coarse adjustment does not completely suppress harmonic content or noise from the power grid. This is done to make evident the dependence of speed estimation using single harmonics with the current filtering, recreating a possible real field case.

In Fig. 6, the spectrogram of the sequence components of the filtered current is shown. The harmonic components used for speed estimation through single tracking are highlighted. In the dashed line, the search band of the maximum amplitudes for each of the harmonics used for speed estimation is displayed $(250 \mathrm{~Hz}, 350 \mathrm{~Hz}$, and $650 \mathrm{~Hz})$. Through this strategy, three estimated rotor speeds are obtained from each specific harmonic. These speeds are plotted in Fig. 8, being named with the frequency used for the estimation. From both Fig. 6 and Fig. 8 it can be seen that the estimator is disoriented at approximately $11.5 \mathrm{~s}$ for the $250 \mathrm{~Hz}$ and $350 \mathrm{~Hz}$ components and at $3.1 \mathrm{~s}$ for the $650 \mathrm{~Hz}$ component.

This results in high error values in the estimation with respect to the direct measurement as seen in Fig. 8. Regardless, it should be noted that the error decreases as speed increases.

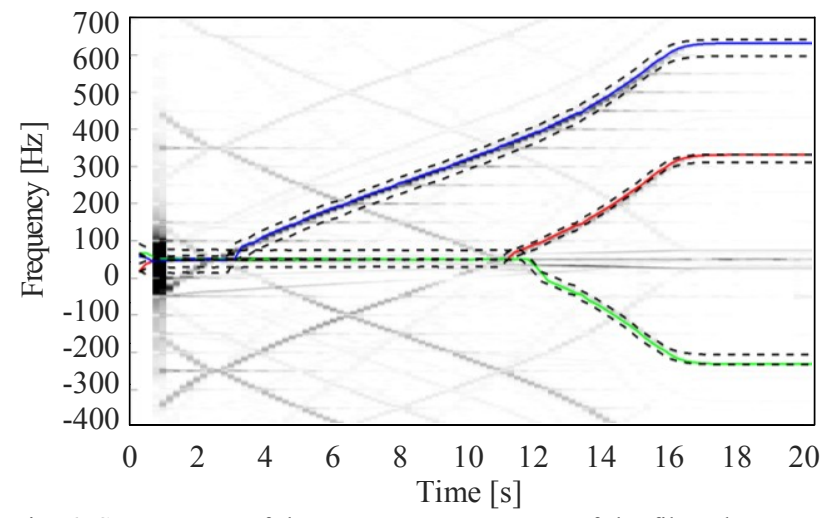

Fig. 6. Spectrogram of the sequence components of the filtered current using single harmonics tracking and coarse adjustment of the comb filter.

\section{Speed estimation using multiple harmonics at reduced voltage starting}

For the analysis of this case study, the same acquisition as the previous case and comb filter settings were used. Fig. 7 shows the spectrogram of the sequence components of the filtered current. The weights assigned to each of the frequency components in (10) were $\alpha_{1}=1, \alpha_{2}=1$ and $\alpha_{3}=2$. Moreover, the harmonic components used in (10) are highlighted. It can be seen that the $650 \mathrm{~Hz}$ harmonic, which is of positive sequence, stands out above the others. For this reason, the $\alpha_{3}$ coefficient is of greater weight. The frequency bands are plotted in dashed line, where the maximum value in amplitude is searched for each harmonic.

Once looking for the maximum according to (12) and applying this value in (5), the speed estimation is obtained for the starting transient indicated as multiple in Fig. 8. Here it can be observed the fulfillment of the confidence criteria established in Section III.F. The measured speed during the starting transient is compared with that estimated from multiple harmonic components of the stator currents full spectrogram, as well as the correlation with the stator current (speed of $1 \mathrm{p} . \mathrm{u}=1500 \mathrm{rpm}$ ). Note that the starting current does not exceed the rated one. This is due to the low voltage used in the IM starting to achieve longer times.

The multiple harmonics tracking strategy correctly estimates the speed during the slow start transient and the error in steady state is less than $0.046 \%$. The error in steady state is of the same order as that reported for other techniques such as those analyzed in Table I.

The main difference between Fig. 6 and Fig. 7 is the way the center frequency is calculated for the search band of maximum amplitudes of the harmonic components. Since the speed estimation using multiple harmonics uses the objective function (10), the center frequency is a weighting of three harmonic components. For this reason, the estimator does not become disoriented or lose track of the harmonics. In the case of speed estimation using single harmonics, when using a harmonic's own estimation to determine its new center frequency, it becomes more susceptible to signal noise and highly dependent on the comb filter design. The robustness of this strategy makes a reliable estimate less dependent on signal conditions.

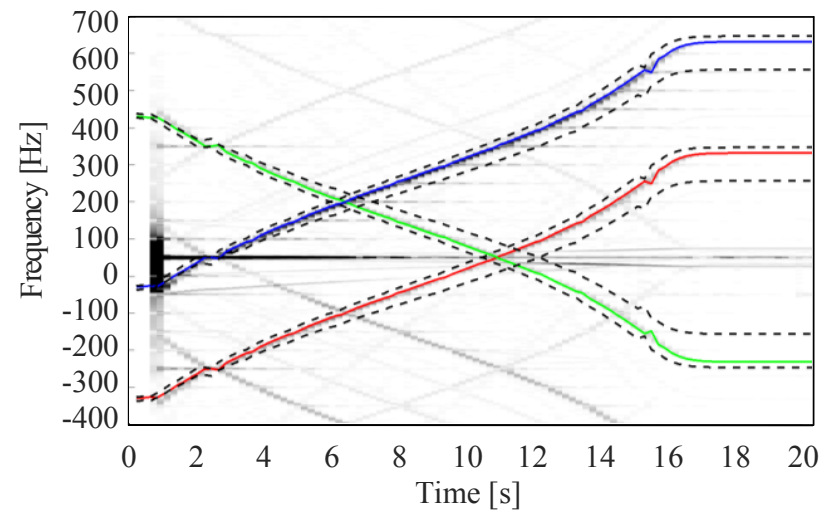

Fig. 7. Spectrogram of the sequence components of the filtered current using multiple harmonics tracking and coarse adjustment of the comb filter. 


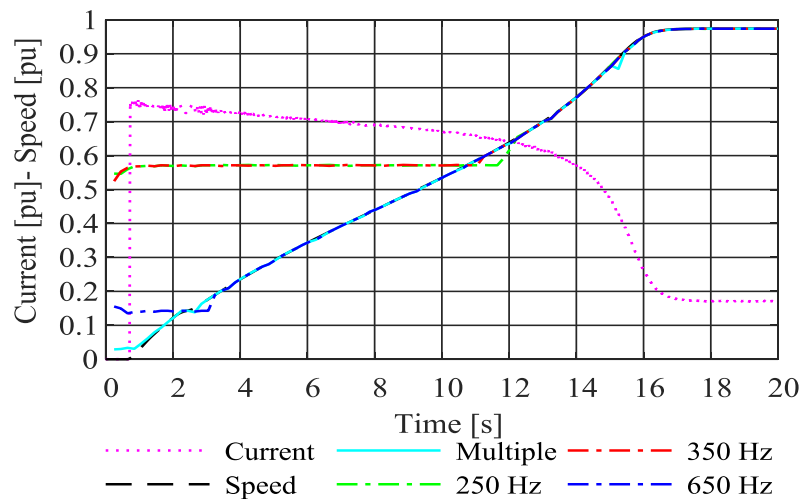

Fig. 8. Estimated speed and measured speed for reduced voltage starting with coarse adjustment of the comb filter.

\section{Speed estimation with star-delta starting}

The star-delta starting, due to its discontinuous characteristic in the connection to the electrical network, presents more severe conditions for speed estimation. As an example, for model-based estimation methods, the change from star to triangle produces an instantaneous change in the IM parameters. For this test condition, the motor was supplied with $70 \mathrm{~V}$ line voltages. The starting begins with the IM connected in star connection and after 18 seconds the starter switches to delta connection. Only the speed estimation by multiple harmonics tracking is analyzed to show the performance of the strategy with another starting condition.

Fig. 9 shows the spectrogram of the sequence components with the followed harmonics and the adaptive search bands. In Fig. 10 it can be observed the estimated speed, the measurement directly, and its correlation with the stator current.

This new proposal is robust to network disturbances, such as a sudden increase in current when switching from star to delta connection. The maximum error is $3.4 \%$ and occurs at the intersections with the comb filter at low speeds and when the connection changes. Then, for other speeds, it remains below $0.2 \%$.

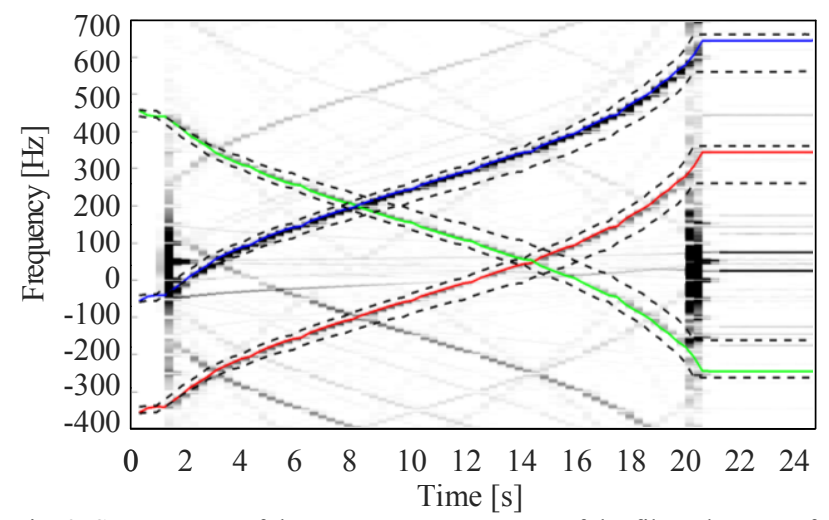

Fig. 9. Spectrogram of the sequence components of the filtered current for star-delta starting using multiple harmonics tracking.

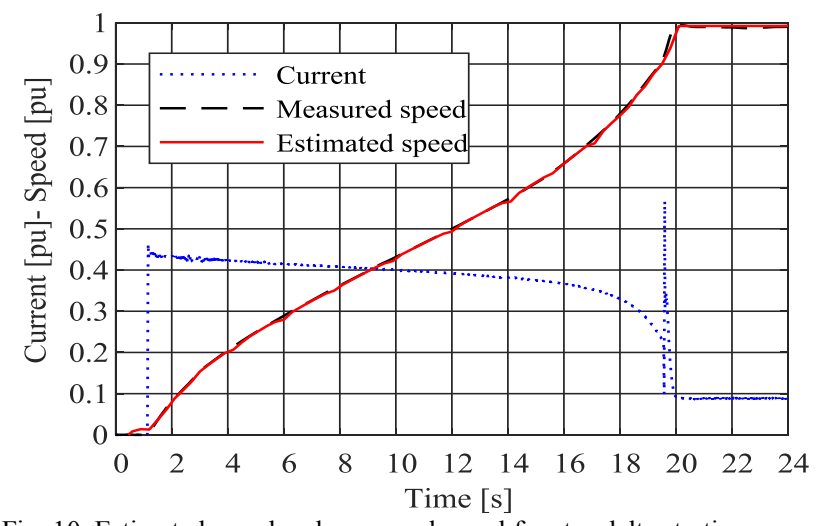

Fig. 10. Estimated speed and measured speed for star-delta starting.

\section{CONCLUSION}

A novel speed self-sensing strategy was presented, based on the simultaneously tracking of multiple harmonic components of the stator currents full spectrogram. Through experimental laboratory results, it was verified that: i) reliably determines the speed from low speeds to the rated speed; ii) it is hardly sensitive to network variations or signal noise; iii) is essential the signal processing to be analyzed for the reduction of errors in the estimation; iv) the definition of the parameters of the filters and the spectrogram windows quickly converges to the solution with a small number of tests; and v) the use of the STFT for time-varying interharmonics analysis is enough for the defined scope [17].

The major contributions of this work included: i) an estimation less sensitive to measurement noise and tracking errors caused by other harmonic components that are not linked to the rotor speed; ii) a simple strategy to understand and customize (signal processing); and iii) a strategy independent of motor parameters, rotor type and presence of fault-related harmonic components.

This new approach significantly improves the performance and reliability of speed estimation during the starting transient of induction motors without the need for motor-specific parameters. It aims to diagnose problems in starters, coordinate protections, or switching times.

\section{ACKNOWLEDGMENT}

This research was supported by the Faculty of Engineering of the UNCPBA; the Faculty of Engineering of the UNRC; the ANPCYT; CONICET and the CIC-PBA.

\section{REFERENCES}

[1] M. Haji, S. Ahmed and H. A. Toliyat, "Induction machines performance evaluator 'torque speed estimation and rotor fault diagnostic'," APEC. Seventeenth Annual IEEE Applied Power Electronics Conference and Exposition (Cat. No.02CH37335), Dallas, TX, USA, 2002, pp. 764-769 vol.2.

[2] M. Meira, G. Bossio, F. Gachen, J. María Bossio, C. Ruschetti and C. Verucchi, "Fault Detection in Starter Resistor of Large Wound Rotor Induction Motor: a Case Study," 2019 XVIII Workshop on Information Processing and Control (RPIC), Bahía Blanca, Argentina, 2019, pp. 7681.

[3] G. Wang, M. Valla and J. Solsona, "Position Sensorless Permanent Magnet Synchronous Machine Drives-A Review," in IEEE Transactions on Industrial Electronics, vol. 67, no. 7, pp. 5830-5842, July 2020. 
[4] Y. Zhang, Z. Yin, Y. Zhang, J. Liu and X. Tong, "A Novel Sliding Mode Observer With Optimized Constant Rate Reaching Law for Sensorless Control of Induction Motor," in IEEE Transactions on Industrial Electronics, vol. 67, no. 7, pp. 5867-5878, July 2020.

[5] J. Chen and J. Huang, "Alternative Solution Regarding Problems of Adaptive Observer Compensating Parameters Uncertainties for Sensorless Induction Motor Drives," in IEEE Transactions on Industrial Electronics, vol. 67, no. 7, pp. 5879-5888, July 2020.

[6] O. C. Kivanc and S. B. Ozturk, "Sensorless PMSM Drive Based on Stator Feedforward Voltage Estimation Improved With MRAS Multiparameter Estimation," in IEEE/ASME Transactions on Mechatronics, vol. 23, no. 3, pp. 1326-1337, June 2018.

[7] M. K. Metwaly, N. I. Elkalashy and M. S. Zaky, "Discrete Sine and Cosine Transforms for Signal Processing Spectral Overlap Saliencies of Induction Machine," in IEEE Transactions on Industrial Electronics, vol. 65, no. 1, pp. 189-199, Jan. 2018.

[8] A. Sapena-Bano, M. Riera-Guasp, R. Puche-Panadero, J. MartinezRoman, J. Perez-Cruz and M. Pineda-Sanchez, "Harmonic Order Tracking Analysis: A Speed-Sensorless Method for Condition Monitoring of Wound Rotor Induction Generators," in IEEE Transactions on Industry Applications, vol. 52, no. 6, pp. 4719-4729, Nov.-Dec. 2016

[9] D. Camarena-Martinez, C. A. Perez-Ramirez, Martin ValtierraRodriguez, Juan Pablo Amezquita-Sanchez, Rene de Jesus RomeroTroncoso, "Synchrosqueezing transform-based methodology for broken rotor bars detection in induction motors", Measurement, Volume 90, 2016, Pages 519-525.

[10] Chuan Li, Vinicio Sanchez, Grover Zurita, Mariela Cerrada Lozada, Diego Cabrera, "Rolling element bearing defect detection using the generalized synchrosqueezing transform guided by time-frequency ridge enhancement", ISA Transactions, Volume 60, 2016, Pages 274-284,

[11] C. J. Verucchi, G. G. Acosta and E. M. Carusso, "Influence of the motor load inertia and torque in the fault diagnosis of rotors in induction machines," in IEEE Latin America Transactions, vol. 3, no. 4, pp. 48-53, Oct. 2005.

[12] W. L. Silva, A. M. N. Lima and A. Oliveira, "Speed Estimation of an Induction Motor Operating in the Nonstationary Mode by Using Rotor Slot Harmonics," in IEEE Transactions on Instrumentation and Measurement, vol. 64, no. 4, pp. 984-994, April 2015.

[13] M. Meira, C. Ruschetti, C. Verucchi, J. M. Bossio and G. Bossio, "A Speed Estimation Strategy for Wound Rotor Induction Motor," 2018 IEEE Biennial Congress of Argentina (ARGENCON), San Miguel de Tucumán, Argentina, 2018, pp. 1-8.

[14] K. Hurst and T. G. Habetler, "Sensorless speed measurement using current harmonic spectral estimation in induction machine drives," IEEE Trans. Power Electron., vol. 11, no. 1, pp. 66-73, Jan. 1996.

[15] S. Nandi, S. Ahmed, and H. A. Toliyat, "Performance analysis of a three-phase induction motor under mixed eccentricity condition," IEEE Trans. Energy Convers., vol. 17, no. 3, pp. 392-399, Sep. 2002.

[16] S. Nandi, S. Ahmed, and H. A. Toliyat, "Detection of rotor slot and other eccentricity related harmonics in a three phase induction motor with different rotor cages," IEEE Trans. Energy Convers., vol. 16, no. 3, pp. 253-260, Sep. 2001.

[17] C. Chen and Y. Chen, "Comparative Study of Harmonic and Interharmonic Estimation Methods for Stationary and Time-Varying Signals," in IEEE Transactions on Industrial Electronics, vol. 61, no. 1, pp. 397-404, Jan. 2014.

[18] M. Meira, G. Bossio, C. Verucchi, C. Ruschetti and J. Bossio, "A speed self-sensing method in starting of induction motors," 2019 XVIII Workshop on Information Processing and Control (RPIC), Bahía Blanca, Argentina, 2019, pp. 53-58.

[19] G. M. Joksimović, J. Riger, T. M. Wolbank, N. Perić and M. Vašak, "Stator-Current Spectrum Signature of Healthy Cage Rotor Induction Machines," in IEEE Transactions on Industrial Electronics, vol. 60, no. 9, pp. 4025-4033, Sept. 2013.

[20] C. Pezzani, G. Bossio and C. De Angelo, "Winding distribution effects on induction motor rotor fault diagnosis", Mechatronics, vol. 24, no. 8 , 2014, pp. 1050-1058.

[21] Carvajal, F. A., Ramírez, J. M., Arcos, L. F., "Diagnóstico en línea y fuera de línea de motores de inducción de baja, mediana y alta tensión", Boletín IIE, Marzo-Abril, México (1999).
[22] S. Giaccone, G. Bossio y G. García, "Análisis de las corrientes del motor de inducción con falla en el estator", AADECA 2004 - XIX Congreso Argentino de Control Automático.

[23] G. A. Magallán, C. H. De Angelo, and G. O. García, "Eliminación de Interferencia Armónica para la Detección de Fallas en Motores Eléctricos," Revista Iberoamericana de Automática e Informática Industrial, ISSN 1697-7912, vol. 6, pp. 89-97, Abril 2009.

[24] D. A. Elvira-Ortiz, D. Morinigo-Sotelo, L. Morales-Velazquez, R. A. Osornio-Rios, R. J. Romero-Troncoso, "Non-linear least squares methodology for suppressing the fundamental frequency in the analysis of electric signals", Electric Power Systems Research, vol. 175, Oct. 2019.

[25] Isermann, "Fault-Diagnosis Systems: An Introduction from Fault Detection to Fault Tolerance". Springer, 2005.

[26] P. Goldman, A. Muszynska, Application of Full Spectrum to Rotating Machinery Diagnostics, Orbit, 17-21, Bently Nevada Corporation, USA, 1st Quarter, 1999.

[27] H. Henao, T. Assaf and G. A. Capolino, "The discrete Fourier transform for computation of symmetrical components harmonics," 2003 IEEE Bologna Power Tech Conference Proceedings, Bologna, Italy, 2003, pp. 6 pp. Vol.4-

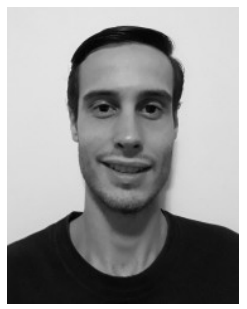

Matias Meira (S'19) received the B.S. degree in electromechanical engineering from UNCPBA, Argentina, in 2016.

$\mathrm{He}$ is currently pursuing a $\mathrm{PhD}$ in engineering in the UNLP, Argentina. His interest in research includes the detection and diagnosis of faults in electric motors and transformers.

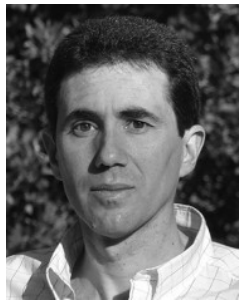

Guillermo R. Bossio (S'03-M'07-SM'15) received the Electrical Engineering degree from UNRC, Argentina, in 1999, and the Doctor of Engineering degree from the UNLP, Argentina, in 2004. His research interests include fault diagnosis of electric machines, electric vehicles, and renewable energy generation.

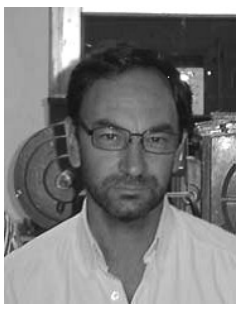

Carlos J. Verucchi received the B.S. degree in electromechanical engineering from UNCPBA, Argentina, in 1994 and the M.S degree in engineering sciences from $\mathrm{UdeC}$, Chile, in 2000 .

$\mathrm{He}$ is currently a professor and researcher in the UNCPBA, in the research group INTELyMEC and in the CIC-PBA.

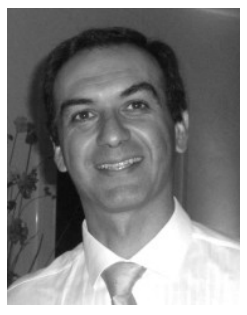

Cristian R. Ruschetti (S'11 - M'13) received the B.S. degree in electromechanical engineering from UNCPBA, Argentina, in 2006 and the $\mathrm{PhD}$ degree in engineering sciences from UNRC, Argentina, in 2012. His research interests are the design and analysis of electrical machines and electromagnetic devices using the FEM.

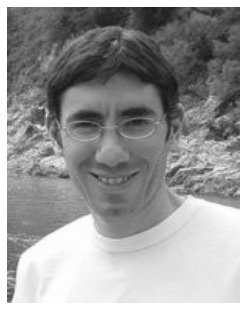

José M. Bossio (S'09 - M'13) received the Electrical Engineer degree and the Magister in Engineering Sciences at the UNRC, Argentina, in 2006 and 2010, and the Doctor in Systems Control degree from the National UNS, Argentina, in 2012. His research interests are the fault diagnosis in electric machines, variable speed drives, and power generation from renewable sources. 